Lösung für Selbstständige kommt

Das Vorhaben, den Mutterschutz auf Studentinnen zu erweitern, war bei Bundesbildungsministerin Johanna Wanka (CDU) zunächst auf großen Widerstand gestoßen. Deshalb lagen die Gesetzespläne monatelang auf Eis. Letztlich verständigte sich die große Koalition aber darauf, dass Ausnahmen von den strengen Mutterschutzregelungen möglich sein sollen - etwa wenn eine schwangere Studentin kurz vor der Entbindung freiwillig eine wichtige Klausur schreiben möchte.
Der Deutsche Gewerkschaftsbund (DGB) hält das Reformpaket für nicht ausreichend. Die stellvertretende DGBVorsitzende Elke Hannack bemängelte, Beamtinnen, Richterinnen und Soldatinnen würden nicht in das bundesgesetzliche Mutterschutzrecht einbezogen. „Das ermöglicht ohne Not Abweichungen vom einheitlichen Schutzstatus.“ Das Familienministerium wies diesen Vorwurf als unberechtigt zurück. Der Mutterschutz für die genannten Berufsgruppen werde zwar aus gesetzestechnischen Gründen in gesonderten Rechtsverordnungen ge- regelt, doch bei der Umsetzung sei der gleiche Schutz gewährleistet wie für alle schwangeren und stillenden Frauen. Die Gewerkschaft Verdi forderte außerdem, neben Angestellten müssten alle erwerbstätigen Frauen in das Mutterschutzgesetz einbezogen werden, nicht nur - wie jetzt vorgesehen - die arbeitnehmerähnlich Selbstständigen.

Ministerin Schwesig versicherte derweil, sie wolle in den kommenden Jahren auch eine Lösung für selbstständige Frauen finden. Dieses Vorhaben werfe allerdings neue Fragen auf. Jana Kötter

\title{
Ärzte und Apotheker einigen sich auf Medikationsplan
}

\section{Der bundeseinheitliche Medikationsplan ist Beschlusssache. Ärzte und Apotheker haben sich auf die Details zum Plan geeinigt, der ab 1. Oktober 2016 allen GKV-Patienten zustehen soll.}

$\mathrm{D}$ er Medikationsplan wird Angaben zum Wirkstoff, zum Handelsnamen, zur Stärke, zur Darreichungsform, zur Dosierung und zur sonstigen Anwendung sowie zum Anwendungsgrund der verschriebenen Arzneimittel enthalten in dieser Reihenfolge (Abb. 1). Dabei sollen sowohl verordnete als auch vom $\mathrm{Pa}-$ tienten in der Apotheke gekaufte Arzneimittel verzeichnet werden. Das sieht die gemeinsam verabschiedete Rahmenvereinbarung von Bundesärztekammer (BÄK), KBV und Deutschem Apothekerverband vor. Anspruch auf einen Medikationsplan haben GKV-Patienten ab 1. Oktober 2016, wenn sie drei oder mehr verordnete Arzneimittel anwenden. Inhalt der Vereinbarung sind auch Vorgaben zur Aktualisierung sowie ein Verfahren zur Fortschreibung des Medikationsplans. Demnach soll eine Arbeitsgruppe regelmäßig überprüfen, wie praktikabel das Verfahren ist.

Die Vertragspartner wollen, dass der Plan ,in einheitlich standardisierter Form umfassend, übersichtlich und patientenverständlich" gestaltet ist. Ärzte, die einen Medikationsplan vom Patienten erhalten, können jedoch nicht sicher sein, dass tatsächlich alle Arzneimittel, die dieser einnimmt, aufgelistet sind, und sollten deshalb auf die Nachfrage beim Patienten selbst nicht verzichten.

Ärzte erhalten für den Medikationsplan eine Vergütung. Der erstausstellende Arzt ist zur weiteren Aktualisierung verpflichtet, Apotheker machen dies auf Wunsch des Versicherten. Aktualisierungen durch andere Ärzte und Kran- kenhäuser sind ebenfalls möglich. Mit dem Medikationsplan sei auch „der Weg für die elektronische Lösung im Rahmen der Telematikinfrastruktur gebahnt", erläutert Dr. Franz Bartmann, Vorsitzender des Ausschusses Telematik der BÄK. Zunächst liegt der Medikationsplan in Papierform vor. Das heißt, der Arzt speichert ihn in seiner Praxissoftware ab und druckt ihn für den Patienten aus. Ab 2018 soll er dann auch auf der elektronischen Gesundheitskarte (eGK) gespeichert werden. Ab dem 1. Januar 2019 müssen dann alle Vertragsärzte und Apotheker in der Lage sein, einen auf der eGK gespeicherten Medikationsplan zu aktualisieren.

Hauke Gerlof

\begin{tabular}{|c|c|c|c|c|c|c|c|c|c|c|c|}
\hline \multicolumn{2}{|c|}{ 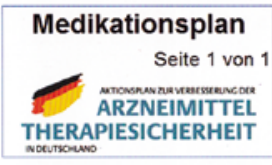 } & \multicolumn{7}{|c|}{$\begin{array}{l}\text { für: Armin Müller } \\
\text { ausgedruckt von: } \\
\text { Apotheke am Sachsendamm } \\
\text { Thüringer Str. 22, 10555 Berlin } \\
\text { Tel.: 030-1234567 } \\
\text { apo-sachsendamm@kbv-net.de }\end{array}$} & \multicolumn{2}{|r|}{$\begin{array}{l}\text { geb. am: 19.10.1959 } \\
\text { druckt am: } 27.03 .2014 \\
\end{array}$} & \multirow[t]{2}{*}{\begin{tabular}{|l} 
\\
\\
\\
Grund
\end{tabular}} \\
\hline \multirow{2}{*}{$\begin{array}{l}\text { Wirkstoff } \\
\text { Metoprololsuccinat }\end{array}$} & \multicolumn{2}{|c|}{ Handelsname } & \multirow{2}{*}{\begin{tabular}{|r|} 
Stärke \\
$95 \mathrm{mg}$
\end{tabular}} & \multirow{2}{*}{\begin{tabular}{|l} 
Form \\
$A A B$
\end{tabular}} & \multicolumn{2}{|c|}{ Mo Mi } & \multicolumn{2}{|c|}{$A b \quad z N$} & \multirow{2}{*}{\begin{tabular}{l|} 
Einheit \\
Stck
\end{tabular}} & \multirow{2}{*}{\begin{tabular}{|l} 
Hinweise \\
Mit 1 Glas Wasser
\end{tabular}} & \\
\hline & $\begin{array}{l}\text { Metopololusccinx } \\
\text { Pharms } 95 \text { ma nets. }\end{array}$ & & & & 1 & 0 & 0 & 0 s & & & Herz/Blutdruck \\
\hline Ramipril & Ramipril-ratiop & oharm ${ }^{\circ}$ & $5 \mathrm{mg}$ & TAB & 1 & 0 & 0 & 0 s & Stck & Mit ausreichend FlUssigkelt & Blutdruck \\
\hline Clopiclogrel & Clopidogrel Ze & entiva ${ }^{0}$ & $75 \mathrm{mg}$ & FTA & 0 & 0 & 1 & $0 \mathrm{~s}$ & Stck & & Herz \\
\hline Pantoprazol & Pantoprazol du & ura $0^{\circ}$ & $20 \mathrm{mg}$ & TMR & 1 & 0 & 0 & $0 \mathrm{~s}$ & Stck & 1 Stunde vor der Mahlzeit & Magen \\
\hline Insulin aspart & NovoRapld ${ }^{\circ} P$ & Penfili & $100 \mathrm{E} / \mathrm{ml}$ & PAT & 20 & 0 & 20 & $\begin{array}{lll}0 & 1\end{array}$ & I.E. & 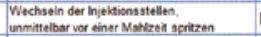 & Diabetes \\
\hline Simvastatin & Simva-Aristo ${ }^{\circ}$ & & $40 \mathrm{mg}$ & FTA & 0 & 0 & 1 & $0 \mathrm{~s}$ & Stck & Mit ausreichend Flüssigkeit & Blutferte \\
\hline Torasemid & Torsamid Hexe & & $5 \mathrm{mg}$ & $T A B$ & 1 & 0 & 0 & $0 \mathrm{~s}$ & Stck & Mit etwas Flüssigkeit & Blutdruck \\
\hline \multicolumn{12}{|c|}{ Zeitlich befristete Medikation } \\
\hline Clarithromycin & \multicolumn{2}{|c|}{ Clarithromycin-TEVA ${ }^{\circ}$} & $250 \mathrm{mg}$ & FTA & \multicolumn{4}{|c|}{ alle 12 Std. 1} & Stck & von 1.4. bis 6.4 & Bronchitis \\
\hline \multicolumn{12}{|c|}{ Selbstmedikation } \\
\hline Myrtol & \multicolumn{2}{|l|}{ GeloMyrtoof } & $120 \mathrm{mg}$ & KPS & 2 & 2 & 2 & 0 s & Stek & 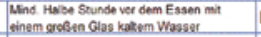 & Bronchitis \\
\hline Johanniskraut & \multicolumn{2}{|l|}{ Laif' Balance } & $900 \mathrm{mg}$ & FTA & 1 & 0 & 0 & $0 \leqslant$ & Stck & Nach dem Frühstuck & Stimmung \\
\hline \multicolumn{12}{|c|}{ Selbstmedikation bei Bedarf } \\
\hline Magnesium & \multicolumn{2}{|c|}{ Magnesium Verla } & $121,5 \mathrm{mg}$ & BTA & \multicolumn{4}{|c|}{ bei Beclarf 1-2 } & Stek & & Wadenkrämpfe \\
\hline Diphenhydramin-HC & 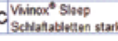 & & $50 \mathrm{mg}$ & $T A B$ & 0 & 0 & $|0|$ & 15 & Stck & 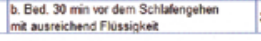 & Schlafstörungen \\
\hline
\end{tabular}

Abb. 1: Muster für den Medikationsplan 